\title{
MODULATION OF INFLAMMATORY RESPONSES BY
}

\section{SUTHERLANDIA FRUTESCENS}

\section{Wei Lei}

\author{
Dr. Kevin L. Fritsche, Dissertation Supervisor
}

\begin{abstract}
Sutherlandia frutescens (L.) R. Br (Lessertia frutescens) is a medicinal plant traditionally used in southern Africa. It has been used for patients suffering from numerous types of cancer, infectious diseases, and various inflammatory conditions. This study was designed to determine the impact of $S$. frutescens on the inflammatory response and anti-microbial activities on cell and/or animal models. Aqueous and ethanolic extracts of $S$. frutescens were made and verified using HPLC. These extracts were used to treat murine macrophages (e.g., RAW 264.7 cells and primary macrophages isolated from mice) to evaluate the impact of $S$. frutescens on in vitro inflammatory responses. This study found that the aqueous extract and a polysaccharide-enriched fraction from the aqueous extract exhibited an immuno-stimulatory activity on murine macrophages. Treatment with aqueous extract or polysaccharides increased the production of reactive oxygen species (ROS), nitric oxide (NO), and inflammatory cytokines/chemokines via activating the toll-like receptor 4 signaling pathway.

On the other hand, the ethanolic extract of S. frutescens dose-dependently decreased the production of ROS, NO, inducible nitric oxide synthase (iNOS), and various inflammatory cytokines and chemokines in murine macrophages co-stimulated with lipopolysaccharide (LPS) and interferon gamma (IFN $\gamma$ ). Follow up experiments
\end{abstract}


demonstrated that the anti-inflammatory activity of the ethanolic extract was mediated via reductions in the activation of $\mathrm{NF}-\kappa \mathrm{B}$, extracellular-signal-regulated kinase $1 / 2$ (ERK1/2), and signal transducers and activators of transcription 1 (STAT1). RNA sequencing provided more evidences to support the anti-inflammatory activity of the ethanolic extract of $S$. frutescens. To our surprise, chlorophylls isolated from S. frutescens had a greater effect on the anti-inflammatory of $S$. frutescens than that of unique compounds (i.e., sutherlandiosides and sutherlandins).

To investigate the impact of oral consumption of $S$. frutescens on in vivo inflammatory responses and anti-microbial activities, mice were fed with AIN-93G based diet with/without containing ground $S$. frutescens powder or were gavaged with $\mathrm{S}$. frutescens extracts followed by challenge with E. coli or LPS. These experiments found that oral consumption of $S$. frutescens had limited or no impact on the in vivo inflammatory responses and anti-microbial activities.

Overall, this study provide a better understanding on the beneficial therapeutic properties of $S$. frutescens using in vitro models, however these studies in a laboratory mouse model suggest that consumption of $S$. frutescens had only a modest impact on host anti-microbial and inflammatory responses to a gram-negative microbial challenge whether intact microbes or bacterial endotoxin (i.e., LPS) was used. 